\title{
MODELO DE EVALUACIÓN DEL DESEMPEÑO DE EMPRESAS INDUSTRIALES Y COMERCIALES DEL ESTADO Y DE ECONOMÍA MIXTA
}

\author{
Bernardo Barona Z., PhD.* \\ Claudia P. Mendieta C., CP. * \\ Carlos Trujillo P., Msc. **
}

\section{INTRODUCCIÓN}

Desde finales de los años noventas la legislación estableció que el gobiemo colombiano tiene posibilidad de disponer de las utilidades que generen las empresas industriales y comerciales del Estado y las de economía mixta (Reglamentación vigente sobre el tema : Decreto 111 de 1995).

La responsabilidad de hacer los estudios técnico-financieros que sirvieran de base a las decisiones que tome el CONPES sobre el porcentaje de las utilidades retiradas de cada empresa con el fin de ser empleado en la satisfacción de otras necesidades financieras del Estado, recayó desde un principio en el Departamento Nacional de Planeación - DNP. Inicialmente, en 1991, y posteriormente en 1995, el DNP recurrio a la Facultad de Ciencias de la Administración de la Universidad del Valle (de aquí en adelante UV) para que le asesorara en el diseño de los modelos financieros necesarios para llevar a cabo satisfactoriamente esta responsabilidad. En este artículo se describe el modelo diseñado por la UV para el DNP. En razón a que el modelo de evaluación de desempeño histórico de cada empresa es bastante similar al descrito en otra parte (Barona y

\footnotetext{
- Facultad de Ciencias de la Administración, Universidad del Valle.

" Facultad de Ingeniería, Universidad del Valle. Enviar comentarios al profesor Barona,Apartado 25360,Cali. Direcciónelectrónica:bbarona@mafalda.univalle.edu.co
} 
Trujillo, 1994) aquí se hará énfasis en el modelo de pronóstico, el cual se elaboray se fundamentaen el modelo de evaluación de desempeño histórico.

\section{EL PROBLEMA A RESOLVER}

El problema puede verse como conformado por dos partes interrelacionadas. La primera hace referencia a la medición y evaluación del desempeño histórico de cada una de las organizaciones del Estado que se desea analizar. La segunda tiene que ver con la evaluación del impacto probable de políticas alternativas de distribución de utilidades sobre las varias dimensiones del desempeño empresarial. El módulo de evaluación del desempeño (primera parte del modelo) se diseñó con el fin de permitiral DNP obtener información sobre el grado en que cada empresa estudiada está:

- Alcanzando los objetivos de supervivencia y crecimiento.

- Haciendo uso eficiente de los recursos a su disposición.

- Alcanzando los otros objetivos que la sociedad le ha fijado.

- Incurriendo en diferentes tipos de riesgo.

El módulo de Pronóstico, por su parte, busca generar información sobre el impacto probable de decisiones alternativas de distribución de utilidades sobre tres dimensiones claves del desempeño empresarial:

- La supervivencia,

- La rentabilidad, y

- El riesgo financiero.

\section{MARCO CONCEPTUAL.S LA EMPRESA COMO SISTEMA. SOCIO-ECONÓMICO ABIERTO Y EL PAPEL DE LAS EMPRESAS DEL ESTADO EN LA SOCIEDAD}

\section{LA EMPRESA COMO SISTEMA ABIERTO}

En el Gráfico No. 1, se representa la empresa como un sistema abierto y también como un sistema de flujos de dinero. Tanto los flujos de ingresos 
(ventas, etc...) como los de egresos (compra de activos, pagos de intereses, etc...) están determinados en gran medida por las decisiones de la gerencia, pero se ven afectadas también, en diferente grado, por factores exógenos tales como los de su entorno inmediato y los de su macroentorno.

El reconocer las varias fuentes de influencia sobre el desempeño empresarial es crucial para instituciones como el DNP, interesadas en el uso eficiente de los recursos por parte de los diferentes sectores de la economía del país. La responsabilidad de la gerencia en el uso eficiente de los recursos es evidente y no se puede soslayar. Sinembargo, varios otros factores por fuera del control de ésta pueden tener en un momento dado impacto importante sobre el desempeño organizacional. La efectividad de varios mecanismos orientados al logro de mayor productividad empresarial, como el uso de remuneración por rendimiento, depende en gran medida de que esto sea apropiadamente tenido en cuenta al momento de planear su utilización.

En la medida en que las empresas operen en entornos desfavorables que inhiban su crecimiento y dificulten el logro de sus objetivos, sólo pueden esperarse razonablemente resultados significativamente favorables a partir de esfuerzos comprensivos, que incluyan tanto la modificación de los factores inhibidores del entorno, como el establecimiento de gerencias profesionales y responsables que den explicación por sus actos y reciban adecuada retribución por la obtención de resultados social y económicamente deseables.

\section{EL PAPEL DE LAS EMPRESAS DEL ESTADO EN LA SOCIEDAD}

Para efectos de esta ponencia, se asumirá que las empresas del Estado tienen un doble propósito en la sociedad: como empresas "industriales, comerciales y de economía mixta" deben operar en tal forma que generen excedentes y produzcan retomos adecuados sobre la inversión realizada; como entidad del Estado, se ęspera que simultáneamente sean un instrumento de la política nacional y, consecuentemente, deban tener la tarea de lograr ciertas metas relacionadas con el desarrollo económico y, en general, el bienestar de los habitantes de un país. Como ejemplos de ello

Cuadernos de Administración No 24 / Universidad del Valle / Enero 1998 


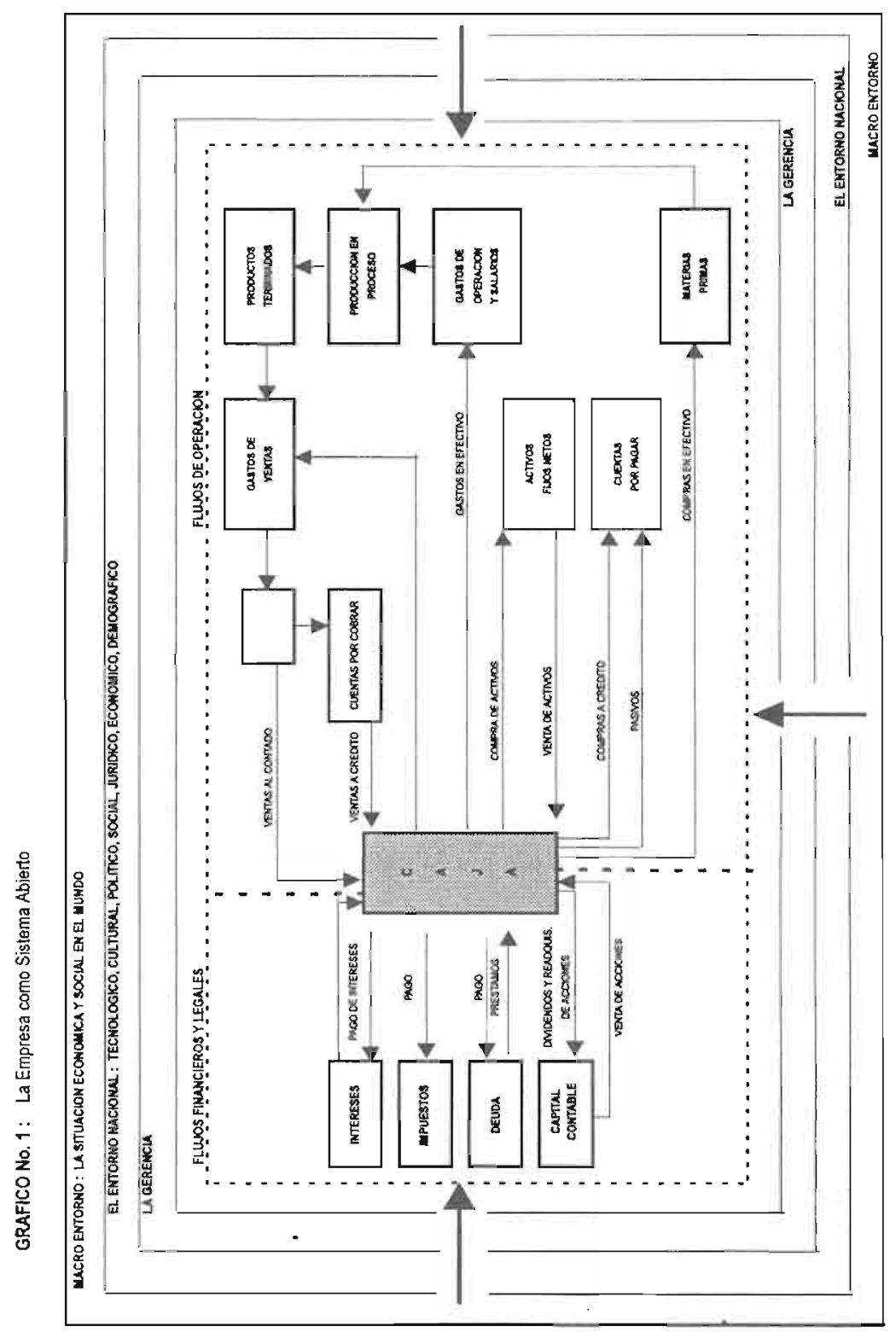

62 Modelo de evaluación del desempeño...Barona Z. /Trujillo P. M Mendieta C. 
tenemos la generación de empleo, la generación de divisas, el desarrollo de independencia técnica y tecnológica del país, etc.

\section{EL MODEL.O DE EVALUACIÓN DEL DESEMPEÑO DE LA UNIVERSIDAD DEL. VALLE - MODELO UV}

\section{MOdELO DE EVALUACIÓN DEL DESEMPEÑO HISTÓRICO}

\section{A. Principios básicos que gobiernan el modelo}

- El objetivo fundamental del modelo es facilitarle al D.N.P. la realización de un diagnóstico global de cada una de las empresas del Estado. El propósito primordial de este diagnóstico, a su vez, es facilitar la toma de decisiones mejor fundamentadas con respecto a la distribución de las utilidades generadas por parte de las empresas del Estado.

- El modelo puede utilizarse también como instrumento para evaluar la gestión de las empresas del Estado, aunque su utilización para este último propósito exigirála realización de algunas modificaciones (por ejemplo, para distinguir cuáles de los resultados de las empresas pueden considerarse como derivadas de las acciones de la gerencia y cuáles no).

- Una empresa del Estado constituye un sistema económico-social abierto. Por consiguiente, su desempeño está influenciado por las acciones de las diferentes personas y grupos que operan al interior de ella, por las decisiones gerenciales y por las fuerzas de su entorno inmediato y de su macroentorno.

- Una empresa del Estado persigue normalmente objetivos múltiples. Como no existe a la fecha ningún indicador individual que permita medir el logro o satisfacción simultáneo de todos ellos, el modelo de medición de desempeño debe hacer uso también de un conjunto múltiple de indicadores.

- Una adecuada comprensión de los factores que se pueden asociar con el desempeño de una empresa no puede obtenerse mediante el examen de los estados financieros (y otras cifras) de ésta para un año especí- 
fico. Para lograr esto, es necesario observar el comportamiento de la empresa durante períodos relativamente largos de tiempo (4 años o más).

- Los informes financieros de las empresas del Estado (basados en la contabilidad) constituyen una fuente importante de indicadores de desempeño. No obstante, varias de las dimensiones del desempeño de una empresa no son medidas adecuadamente por la contabilidad. Por esta razón, es necesario hacer uso, también, de una variedad de indicadores de naturaleza no contable.

- La información sobre los flujos históricos de caja constituyen el insumo escencial para la medición de algunas dimensiones del desempeño de la empresa. La contabilidad de causación, base de los estados financieros (Balance General y Estado de Resultados) que elaboran las empresas del Estado, es útil para la medición de la rentabilidad y para otros aspectos, pero disfraza los verdaderos movimientos de caja ocurridos al interior de la empresa. Por esta razón, se considera que adicionalmente al Balance General y al Estado de Resultados (y a otra información no contable) las empresas del Estado deberían enviar al D.N.P., anualmente, un Estado de Flujo de Caja.

\section{B. Las seis dimensiones del modelo}

Para la construcción del modelo de evaluación del desempeño histórico los autores hicieron una revisión bibliográfica amplia y sostuvieron varias reuniones con funcionarios del DNP. El modelo que finalmente resultó tenía las seis dimensiones que se describen a continuación: 
GRÁFICA N ${ }^{\circ}$ 1: Dimensiones del Modelo UV

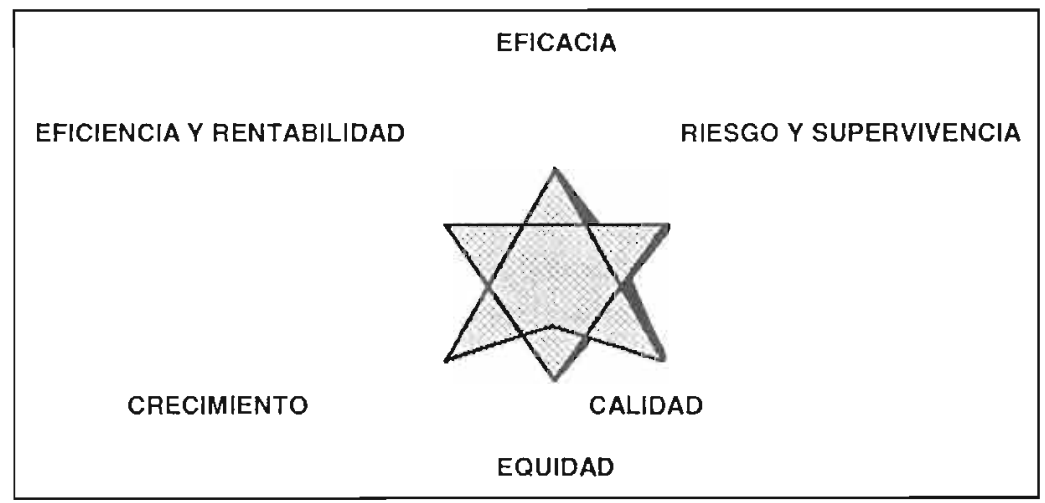

- Eficacia. Ésta se entendió como el grado en que se han alcanzado los objetivos establecidos para la empresa. El modelo UV supuso que un objetivo básico de todas las empresas estudiadas es el de generar valor agregado. Por esta razón, en todos los casos se incluyó el indicador de Valor Agregado Bruto (en \$) generado por la empresa (medido como la diferencia entre las ventas y los costos de todos los materiales y servicios que fueron utilizados en la generación de tales ventas y adquiridos por fuera de la empresa) como un indicador importante de eficacia.

- Eficiencia y rentabilidad. En el análisis financiero de empresas privadas se otorga singular importancia a la evaluación de las utilidades generadas por éstas, ya que constituyen un indicador, tanto de la eficiencia en el uso de los recursos, como de eficacia en el logro de los objetivos últimos para los cuales la empresa fue constituida. En la evaluación del desempeño de empresas del Estado, aunque posiblemente sólo unas pocas personas sostengan hoy en día que la eficiencia no es un factor importante en la evaluación de su desempeño, un número posiblemente más grande sí puede estar. de acuerdo con la afirmación de que existen otras dimensiones que pueden ser más, o al menos, tan importantes como la eficiencia. Esas otras dimensiones son las que en el Modelo UV se han agrupado en la categoría eficacia. 
Los principales indicadores de eficiencia empleados fueron los de rendimiento sobre el Activo y sobre el Patrimonio, productividad del capital y de la mano de obra, y costos unitarios de producción.

Riesgo y supervivencia. La capacidadde supervivencia de una empresa privada depende estrechamente de su habilidad para cancelar las obligaciones (laborales, financieras, con proveedores, etc.) a medida que éstas se van venciendo. Esto conduce al análisis de liquidez corrientemente trabajado en los textos sobre análisis financiero. No debe pasarse por alto, sinembargo, que la supervivencia para las empresas del Estado, depende en menor grado de los indicadores clásicos de liquidez que la empresa privada, esto en razón al respaldo financiero que el Estado tradicionalmente le brinda a sus empresas.

Algunos de los indicadores manejados en esta categoría fueron: razón corriente, prueba acida, razón flujos de caja operativo/intereses, reservas comprobadas de crudo, (para el caso de Ecopetrol) etc.

Es de común aceptación que una completa evaluación del desempeño de una empresa debe relacionar la rentabilidad alcanzada con el riesgo que la empresa está asumiendo en la generación de ésta, por lo tanto, los autores consideraron la medición y evaluación del riesgo como un elemento central en el modelo.

El modelo analiza el riesgo financiero utilizando la relación Pasivo Total/Activo Total (a más alto pasivo total, mayores costos fijos y mayor variabilidad de las ganancias de los propietarios de la empresa). Como indicador del riesgo operacional se utilizó un índice de la variabilidad de las ganancias operacionales durante los pasados cuatro años.

Crecimiento. El crecimiento permite hacer frente a las demandas originadas en el entorno de la empresa (por ejemplo, demanda sobre la generación de energía eléctrica, mayores volúmenes de exportación, etc.) y, a su vez, puede traer beneficios de otra índole, tales como obtención de menores costos de producción, alcance de economías de escala, etc. 
Los principales indicadores de crecimiento que se utilizaron fueron la tasa anual compuesta de crecimiento de las utilidades durante los últimos cinco años y la tendencia de las ganancias en los últimos tres años.

- Calidad. En la literatura especializada se viene considerando desde hace más de una década la calidad como uno de los determinantes fundamentales del desempeño de una empresa. Más aún, algunos autores distinguidos consideran que hoy en día la calidad, más que una ventaja competitiva -como lo fue en la década de los años ochentas- es una necesidad competitiva (Kaplan and Norton, 1996: 87). El Modelo UV incluye algunos indicadores indirectos de calidad (o indicadores de naturaleza "negativa", como algunos los llaman por indicar la presencia de eventos o productos que no deberían haberse producido). Los indicadores de calidad empleados variaron entre los distintos sectores económicos estudiados e incluyen índices de daños y tiempo promedio fuera de servicio.

- Equidad. Esta dimensión es incluida por gran parte de la literatura económica como una de las dos categorías más relevantes para medir el desempeño de un sistema económico (la otra es eficiencia). A pesar de esto la operacionalización de este concepto es muy problemática. Como guía para esto los autores adoptaron el concepto de que una "distribución es equitativa si es el resultado de individuos informados que escogen sólo conjuntos iguales" (Le Grand, 1991:87).

\section{Los elementos constitutivos del modelo}

Éstos son los insumos, el procesamiento electrónico y los informes generados.

- Insumos. Los estados financieros, específicamente el Balance General, el Estado de Resultados y el Estado de Flujos de Caja, tienen un significado en el momento en que empiezan a ser comparados a través del tiempo con empresas similares y con estándares ideales, ya sea en forma bruta o procesada en forma de indicadores. 
El estudio utiliz6́ los formatos originales empleados por las empresas para presentar sus informes, modificados, en parte, por los Ajustes por Inflación y, por otra, por las disposiciones de la Dirección General de la Contabilidad en su Plan General de Conrabilidad Pública. Para el caso especial de los Flujos de Caja, el Modelo UV diseño un formato especial para unificar su formulación.

En relación con la otra información, ésta incluye cifras del número total de empleados, inflación anual, número de clientes, etc.

- El procesamiento de los insumos. El Modelo UV fue desarrollado inicialmente (en 1992) en Lotus 123, pero posteriormente fue actualizado en Excel en su versión de libro. En éste, cada hoja del libro por empresa posee las estructuras de los estados financieros básicos (Balance General, Estado de Resultados y Flujos de Caja); también forman parte del libro, como se verá más adelante, los cálculos intermedios que sirven de base para la elaboración de los pronósticos de los futuros Flujos de Caja de la empresa, bajo varias situaciones alternas de distribución de utilidades y los pronósticos mismos.

- Los informes generados. Para cada empresa y para cada uno de los años estudiados (idealmente los últimos cinco) el modelo calcula una serie de porcentajes e índices, los cuales se presentan agrupados en las cinco categorías que se describen a continuación:

- Análisis de la composición porcentual del activo corriente y del pasivo corriente.

- Otros indicadores de liquidez y de supervivencia en el corto plazo.

- Análisis de rentabilidad y generación de utilidades.

- Indicadores básicos de eficiencia, eficacia, calidad y otros que utilizan información no incluida en los estados financieros.

La hoja resumen es sin duda, el rasgo distintivo y más destacado del modelo UV Ésta se diseñó teniendo en cuenta las necesidades de información de los funcionarios del gobierno, con responsabilidad de tomar decisiones sobre la distribución de las utilidades de las empresas del Estado, del más alto nivel (jefatura del Departamento de Inversiones y Finanzas Públicas del DNP, funcionarios del CONFIS, etc.). 
En la hoja resumen se condensa la información que se considera más importante para la medición de las seis dimensiones del desempeño de las empresas del Estado. También, para aquellos sectores en los que existe un número relativamente grande de empresas (por ejemplo, empresas del sector eléctrico) en la hoja resumen se presenta numérica y gráficamente la comparación que hace el programa de los índices de una empresa específica con las del resto de empresas del sector y el cómputo que se realiza del escalafón o "ranking" de cada empresa basado en la medida estadística de los percentiles, en lugar de otros indicadores que suponen cierta distribución normal en los resultados analizados. Finalmente, la hoja resumen provee un espacio para que se indique el resultado de la "Evaluación global e integral de la empresa". En la realización de ésta se debe tener en cuenta, no solo la comparación de los índices de una empresa con los índices de sus pares (realizada automáticamente por el Modelo UV), sino también el contraste de los índices del último año con los índices de los últimos cuatro años y, también con índices ideales o estándares.

\section{EL MODELO DE PRONOSTICO}

\section{A. Principios básicos que gobiernan el modelo}

- El principal objetivo del Modelo UV de Pronóstico radica en generar información sobre el impacto probable que tendrá en la empresa, objeto del análisis, la decisión de transferir al Estado un determinado porcentaje de las utilidades generadas por ésta.

- El Modelo UV de Pronóstico escogido, corresponde al del Porcentaje de Ventas olngresos, en el cual, se toman los ingresos como la materia prima esencial.

Para pronosticarlos, se analiza la serie de tiempo de los valores alcanzados por éstos en los últimos años (idealmente los últimos $5 \dot{6}$ 10) y mediante un análisis lineal, exponencial o logarítmico se pronostica el volumen de ingresos para el año inmediatamente siguiente al 
último que se tiene disponible. En EXCEL este análisis se hace automáticamente (tanto los cálculos, como el pronóstico).

- A una cantidad de Ingresos o Ventas, corresponde un nivel determinado de recursos que, calculados como demanda, afectan la estructura general de la organización estudiada y deben ponderarse entre un financiamiento extemo (Pasivo Corriente - Pasivo a Largo Plazo) y un financiamiento interno (Utilidades Retenidas).

- Aunque este modelo es de gran aceptación y uso en el ámbito financiero, no debe olvidarse que el conocimiento general del entorno que rodea a cada organización estudiada es pieza clave para el análisis de los resultados obtenidos en cada una de las cinco opciones de distribución de utilidades. Asímismo, el conocimiento y manejo contable-financiero para el análisis de los efectos de la inflación y la influencia de otros rubros en la capitalización (o descapitalización) de las empresas y el sector, son elementos que debe incorporar el analista en sus recomendaciones de Distribución de Excedentes.

- Aunque es cierto que, comoempresas del Estado, toda entidad estudiada por el Modelo UV de Pronóstico debe contribuir economicamente para el desarrollo de los planes sociales propuestos por el gobierno nacional, no debe olvidarse que en un largo plazo son ellas las directamente afectadas (o beneficiadas) por la distribución racional que se realice de sus excedentes; por lo tanto, es necesario tener en cuenta las diferentes políticas que el gobierno defina para los sectores y la decisión asumida en el caso de algunas empresas específicas, a fin de evitar con ello soluciones de coyuntura que no contribuyan en nada al desarrollo futuro de ellas, del sector y del país.

\section{B. Los supuestos fundamentales del Modelo}

- El modelo de pronóstico que se escogí́ es el conocido con el nombre de Porcentaje de Ventas o Ingresos. Este modelo se basa en el supuesto de que todas las partidas de Activos, Pasivos y Gastos varían en proporción directa con el volúmen de Ventas o Ingresos. Éste es el 
"Factor crítico de pronóstico", porque de éste dependerán los valores de la mayor parte de los rubros que se pronostican.

- Una vez se han pronosticado las ventas del año en el que se está haciendo el análisis, se pronostican los recursos financieros y las fuentes disponibles que serán necesarios para dar soporte al cambio esperado en las ventas (Pronóstico de la DEMANDA de recursos). Para hacer estos pronósticos se supone que no se harán nuevos aportes de capital a la empresa y que las únicas fuentes de recursos que estarán disponibles serán las “espontáneas" (al aumentar las ventas, de manera espontánea aumentan los pasivos), las utilidades generadas y retenidas en la empresa y, el financiamiento externo adicional requerido, (diferencia entre la demanda total de recursos y la suma de las fuentes espontáneas y las utilidades retenidas).

- El Modelo UV supone, además, que el ajuste al endeudamiento que se hace como resultado del financiamiento externo adicional (requerido o sobrante) afecta sólo el Pasivo Corriente.

- Este modelo supone, también, que muchas de las relaciones que históricamente se han mantenido hasta el momento del análisis perdurarán durante el periodo de pronósticos.

- Finalmente, el ModeloUV supone cinco escenarios de análisis diferentes: En el primero se supone que no habrá transferencia de recursos al Estado: todas las utilidades que genera la empresa se dejan para uso de ésta. Bajo el segundo escenario, se transfieren al Estado el 20\% de las utilidades generadas por la empresa y así sucesivamente hasta el quinto escenario, en el que el 100\% de las utilidades se transfieren al Estado.

\section{Los elementos constitutivos del modelo \\ El Modelo UV de pronóstico se encuentra conformado por tres módulos generales a saber:}

- Un primer módulo de cálculos intermedios cuya finalidad es la de pronosticar los ingresos del año siguiente con base en los niveles de ventas del año inmediatamente anterior, al igual que una serie de 
razones financieras intermedias necesarias para la realización de los cálculos de pronósticos de los módulos dos y tres.

Algunos de los cálculos realizados en este módulo son, entre otros, razón Ingresos/Activos Corrientes, Utilidad Pronosticada y PasivosTotales/Activos Totales, entre otros.

- El segundo módulo, analiza con base en el pronóstico anterior de Ingresos por Ventas la posible Demanda de Recursos y sus respectivas "fuentes de financiación, según sea el caso, para cada una de las cinco opciones de distribución de utilidades $(0 \%, 20 \%, 50 \%, 80 \%, 100 \%)$. Estas opciones pueden fácilmente cambiarse a fin de trabajar los porcentajes requeridos por el criterio del analista.

Para estimar la demanda de recursos se analiza la cifra de cambio pronosticado de ingresos (Ingresos estimados - Ingresos año anterior) y los promedios en los últimos tres años de las razones: Ingresos/ Activos Corrientes e Ingresos/(Activos Totales - Activos Corrientes). La demanda total de recursos es la suma de las demandas para financiar los dos tipos de activos: Activos Corrientes y Activos No Corrientes.

Con respecto a las "fuentes de financiación", el Modelo UV de pronóstico supone que éstas sólo son tres:

Fuentes espontáneas de crédito, que analizan los cambios sufridos por los Ingresos pronosticados en relación con las razones: Pasivos Corrientes/Ingresos y Pasivos No Corrientes/Ingresos.

Utilidades retenidas, que analizan los Ingresos pronosticados respecto al márgen neto del último año afectado por el factor $1-t_{i}$, donde $t_{i}$ corresponde a la tasa de distribución de utilidades; esta tasa varía según cada una de las cinco opciones consideradas en el Modelo.

Financiamiento externo adicional, el cual resulta de la resta de la Demanda Total de Recursos de las Fuentes Espontáneas de Crédito y las Utilidades Retenidas.

- El tercer y último módulo, presenta un resumen de las posibles utilidades que se transferirán al Estado bajo cada una de las cinco opciones, y presenta un Análisis de la Situación Financiera Resultante 
bajo cada opción. Además de los grandes rubros del Balance General y de la cifra de Excedentes Financieros que resultarían si se cumplen los supuestos de pronóstico, en esta sección se muestran los valores que asumirían cuatro índices financieros que son importantes en materia de Rentabilidad (Rentabilidad sobre el Patrimonio), Riesgo (Razón Pasivo Total/Activo Total y Razón Corriente) y Crecimiento (Tasa de Crecimiento Sostenible).

\section{APORTES Y LIMITACIONES DEL MODEL.O UV}

\section{MODELO DE EVALUACION DEL DESEMPEÑO HISTÓRICO}

El Modelo UV hace utilización plena de las ventajas reconocidas que tiene el uso del computador en el manejo de la información: la facilidad de almacenar y manipular grandes volúmenes de datos y de hacer diversas operaciones matemáticas a grandes velocidades.

El uso apropiado de los modelos desarrollados, sinembargo, requiere de una clara comprensión y de los beneficios e inconvenientes inherentes al modelo en sí y a la utilización de modelos computarizados de análisis financiero.

- Aportes. Los principales aportes fueron, entre otros:

- El relacionar el análisis económico-financiero moderno con los grandes objetivos de las empresas (supervivencia, eficacia, etc.). Esto aunque parezca obvio, es algo que no se hace en la mayor parte de la literatura sobre análisis financiero.

- El proporcionar de manera automática la comparación de una empresa con las del resto del sector.

- Suministro de una base de datos computarizada y amplia, la cual se puede ir actualizando fácilmente para diversos fines.

- El empleo de una herramienta computacional de uso generalizado y de fácil aprendizaje como es Excel. 
- Limitaciones. Las más importantes son, en opinión de los autores, las siguientes:

- El alcance limitado de la información incluida (por esta razón se recomienda guardar los informes escritos a fin de aclarar algunas dudas sobre el origen de la misma).

- Las deficiencias de la información financiera que se utilizó como insumo importante en los modelos. A pesar de que la informacion elaborada por las empresas ha venido mejorando en los últimos años, aún la información del análisis financiero, es muy deficiente.

\section{MODELO DE PRONÓSTICO FINANCIERO}

\section{Aportes}

Con el Modelo UV de Pronóstico pueden lograrse análisis más detallados en cuanto a la estructura económico-financiera de cada una de las empresas analizadas y, de igual forma, emitir un concepto más estructurado y racional acerca de la proporción en que la distribución de utilidades propuesta puede o no afectar a dicha organización.

El Modelo, además, realiza un aporte técnico especial en cuanto a que para el análisis de las tendencias de crecimiento de los ingresos utiliza funciones matemáticas, lógicamente sustentadas que permiten minimizar el factor subjetivo empleado en algunos casos como factor de decisión.

\section{Limitaciones}

Este Modelo presenta algunas limitaciones en cuanto a la forma en que resume o recopila la información contable bajo los rubros provistos por el Plan General de Contabilidad Pública y es por ello que se sugiere que las fuentes iniciales permanezcan al alcance del analista interesado puesto que son éllas las únicas que permiten definir eficientemente el entorno en el cual se desarrollaron y de- esta forma entender los resultados arrojados por cada empresa bajo las políticas manejadas hasta ese momento por el gerente de turno. 
El Modelo UV de Pronóstico, como toda abstracción de una realidad, posee ciertas restricciones (o supuestos), en cuanto al comportamiento de los diferentes rubros contable-financieros acorde con el crecimiento o disminución de los Ingresos; comportamiento que no refleja el ambiente real de la organización pero que, para efectos de un resultado aproximado de la realidad, se deben asumir como ciertos y generalizadores.

El entorno totalmente variable al que se encuentran expuestas actualmente las empresas del Estado, hace que las políticas gubernamentales varíen casi totalmente de un año a otro y, por ello, lo que el modelo puede pronosticar como excedente de recursos, puede llegar a ser realmente un faltante de ellos, puesto que el entorno condiciona enormemente el comportamiento de la organización y, así mismo, sus resultados.

\section{CONCLUSIONES}

Con este trabajo se ha querido ilustrar cómo la aplicación de conceptos financieros modernos utilizando las facilidades que permite el desarrollo actual de la informática puede contribuir a obtener un mayor conocimiento del desempeño de las empresas del Estado y de las implicaciones probables de las decisiones que se tomen sobre la distribución de sus utilidades. La mejor información generada por los modelos aquí descritos puede conllevar, consecuentemente, a una más eficiente utilización de los recursos por parte de la sociedad en su conjunto.

No puede entenderse que el Modelo UV, presentado con sus dos módulos, es algo completamente acabado que resuelve a entera satisfacción el problema que originó su desarrollo. El alcanzar esta meta sólo será posible mediante la realización de un esfuerzo amplio de investigación que incluya, no solo el seguimiento de los modelos de pronóstico desarrollados, su comparación con los flujos de caja que reaimente se dan en la práctica y el correspondiente ajuste después de analizar la comparación, sino, también, la búsqueda e implementación de una mejor reglamentación sobre la forma en que la información financiera de las empresas del Estado debe ser recolectada, procesada y reportada. 


\section{BIBLIOGRAFÍA}

ARRIETA, C. G. y otros (1992). Control y Evaluación de la Gestión Pública. Los desafíos de la nueva Constitución. Departamento Nacional de Planeación. Santa Fe de Bogotá.

BARONA, B. (1986). Distribución de Frecuencia de las razones financieras de Sociedades Anónimas colombianas. Evidencia Empírica. Comisión Nacional de Valores. Boletín, Año 4 Vol.5, diciembre.

BARONA B. y TRUJILLO C. (1994). "Modelo de evaluación del desempeño de empresas industriales y comerciales del Estado". En: J.Cárdenas y W.Zambrano (Editores). Gerencia Pública en Colombia. Consejería Presidencial para la Modernización del Estado. Asociación Colombiana para la Modemización del Estado. Santa Fe de Bogotá.

BEAVER, W. (1981). Financial Reporting: An Accounting Revolution. Prentice Hall. Englewood Cliffs. N.J.

BEETON, D. (Editor) (1988). Performance Measurement. Getting The Concepts Right. Public Finance Foundation. Discussion Paper 18. London.

BERNSTEIN, L.A. (1989). Financial Statement Analysis. Theory, Application, and Interpresation. (Fourth Edition). Irwin, Homewoods, Illinois.

BIOSCA, Rodes J. "La Contabilidad de Excedentes, Líneas Generales" en Cárdenas J.H. y otros 1992. Empresa Pública; control de gestión y evaluación de resultados. Universidad Nacional de Colombia. Facultad de Ciencias Económicas. Programa de Educación Continuada. (Mimeo). Santa Fe de Bogotá. Abril.

BREALEY, R. And S. MYERS (1987) Principles of Corporate Finance. (3rd. edn). McGraw-Hill. New York.

CÁRDENAS, J.H. (1990). Las Empresas Públicas en el proceso de Reforma del Estado en Colombia. Documento preparado para la Comisión Presidencial para la Reforma de la Administración Pública del Estado Colombiano. (Mimeo) Bogotá, abril 6.

DEPARTAMENTO NACIONAL DE PLANEACION (1992). Distribución de los Excedentes Financieros de las Entidades Públicas. Anexo 1. Empresas industriales y comerciales. Documento DNP-2602-UIP.Santa Fe de Bogotá, junio 30 .

FERNÁNDEZ, P. (1986). Managing Relations Between Goverment and Public Enterprises. Internacional Labour Office Geneva.

FOSTER, G. (1986). Financial Statement Analysis. (Second Edition). PrenticeHall, Englewood Cliffs, N.J.

GANDHI, D.K. And T. KOPLYAY (1991). Public Entrerprise Performance measures and Reporting. Ponencia presentada en la XXVI Asamblea de 
Comité Latinoamericano de Escuelas de Administración - CLADEA. Lima, septiembre.

HENDERSON, J. (1992). Against the Economic Orthodoxy: on the Making of the eust asian miracle. Working Paper No.230, Manchester Busines School, Manchester, August.

JONES, L.P. (1991). Performance Evaluation for State Owned Enterprises en Ramamurti, R. and R. Vemon (editors), Privatization and control of state owned enterprises, Economic Development Institute, The World Bank, Washington, D.C.

KAPLAN, R.S. and D.P. NORTON (1996). Translating Strategy into action: The Balanced Scorecard, Harvard Business School Press, Boston.

LEGRAND, J. (1991). Equity and choice, an Essay in Economies and Applied Philosophy: Harper Collins. London.

MANESS, T.S. and HENDERSON, J.W. Financial Analysys and forecasting software system. Prentice Hall International, 1991, chapter 4.

MARTÍNEZ, A. "A mbiro de aplicación del excedente de la productividad global y las cuentas del excedente" en Cárdenas J.H. y otros (1992). Empresa Públicu, Control de Gestión y Evaluación de Resultados. Universidad Nacional de Colombia. Facultad de Ciencias Económicas. Programa de Educación Continuada. Santa Fe de Bogotá. Abril (mimeo).

PARK, Y.C. (1986). A System for Evaluating the Performance of Goverment. Inverted Enierprises in the Republic of Korea. World Bank Discusion Papers No. 3.

POWELL, V. (1987). Improving Public Enterprise Perfonnance. Concepts and Techniques. Intemational Labour Office. Management Development Series No. 22. International Labour Office Geneva.

RAPPAPORT, A. (1986). Creating Shareholder Value The New Standards for Business Performance. The Free Press, New York.

REVISTA DE PLANEACIÓN Y DESARROLLO. Vol. XIX, Números 3 y 4. Diciembre de 1987. Departamento Nacional de Planeación. Bogotá.

SHIRLEY, M.M. (1991). Evaluating the Performance of State Owned Enterprises in Pakistan. En Ramamurti R. and R. Vernon (Editors),

Privatization and Control of State Owned Enterprises. Economic Development Institute, The World Bank, Washington D.C.

. (1989). The Reform of State-Owned Enterprises. Lesson from World Bank Lending. The World Bank. Policy and Research Series. Washington.

WESTON, J. Fred and COPELAND, Th. E. Finanzas en Adminstración Novena Edición. McGraw Hill, Bogotá D.C. 1995 p.p. 303 a 323. 
THE WORLD BANK, COUNTRY ECONOMICS DEPARMENT (1991). The Reform of Public Sector Management. Lessons from Experience. Policy and Research Series No. 18 The World Bank, Washingion D.C.

TURK, I. (1984). Accounting Analysis of the Efficiency of Public Enterprises. International Center for Public Enterprises in Developing Countries. ICPE Monograph Series, No. 19, Ljubljana, Yugoslavia. 